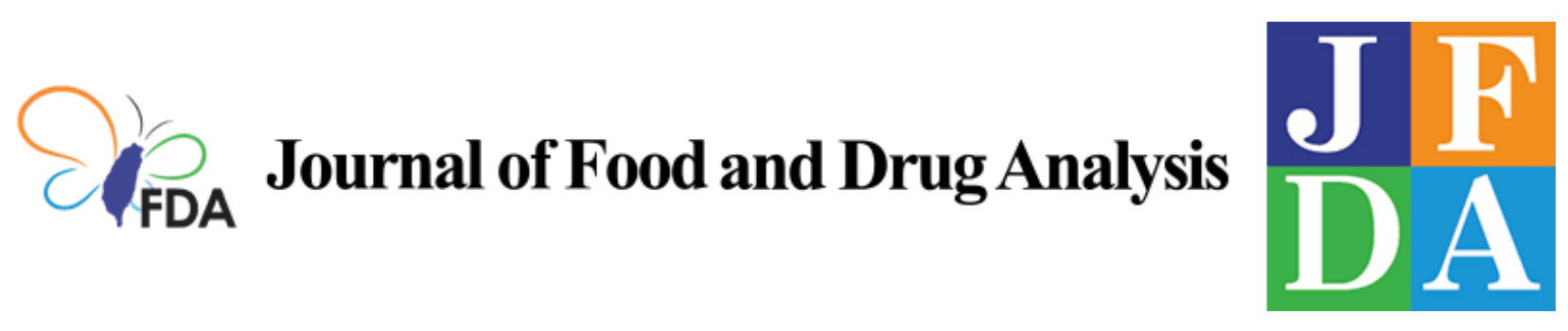

Volume 29 | Issue 4

Article 1

2021

\title{
Recent developments in sensing of oversulfated chondroitin sulfate in heparin. A review .
}

Follow this and additional works at: https://www.jfda-online.com/journal

Part of the Food Science Commons, Medicinal Chemistry and Pharmaceutics Commons, Pharmacology Commons, and the Toxicology Commons

(c) (i) $(9)$

This work is licensed under a Creative Commons Attribution-Noncommercial-No Derivative Works 4.0 License.

\section{Recommended Citation}

Madhu, Manivannan and Tseng, Wei-Lung (2021) "Recent developments in sensing of oversulfated chondroitin sulfate in heparin. A review .," Journal of Food and Drug Analysis: Vol. 29 : Iss. 4 , Article 1. Available at: https://doi.org/10.38212/2224-6614.3379

This Review Article is brought to you for free and open access by Journal of Food and Drug Analysis. It has been accepted for inclusion in Journal of Food and Drug Analysis by an authorized editor of Journal of Food and Drug Analysis. 


\title{
Recent developments in sensing of oversulfated chondroitin sulfate in heparin. A review
}

\author{
Manivannan Madhu ${ }^{a}$, Wei-Lung Tseng ${ }^{a, b, *}$ \\ a Department of Chemistry, National Sun Yat-sen University, No. 70, Lienhai Rd., Kaohsiung, 80424, Taiwan \\ b School of Pharmacy, College of Pharmacy, Kaohsiung Medical University, No.100, Shiquan 1st Rd., Kaohsiung, 80708, Taiwan
}

\begin{abstract}
Oversulfated chondroitin sulfate (OSCS), a non-natural sulfated glycosaminoglycan, recognizes as a significant containment in the pharmaceutical heparin, and it could trigger adverse reactions. Chromatography-, electrophoresis-, electrochemistry-, and spectroscopy-related techniques are currently available for accurate and precise analysis of a trace amount of OSCS in heparin. Recently, emerging studies focus on developing colorimetric and fluorescent probes to monitor OSCS containments in heparin. Therefore, this current review aims to describe the sensing principle and procedure of the reported probes that are sensitive and selective toward OSCS in heparin without the interferences of other sulfated glycosaminoglycans. The reported OSCS-specific probes are comprehensively discussed according to the recognition elements of OSCS, including coralyne, AG73 peptides, positively charged tetraphenylethene derivatives, polythiophene polymer, and poly-L-lysine, protamine, superpositively charged green fluorescent proteins, and poly (diallyldimethylammonium chloride). The sensing of OSCS in heparin is generally achieved using, (i) the specific affinity of the recognition element with OSCS and heparin, (ii) heparinase-mediated hydrolysis of heparin, and (iii) OSCSinduced inhibition of heparinase activity. Additionally, coralyne-based DNA probes can detect OSCS in heparin in the presence of $\mathrm{Ca}^{2+}$ ions without the assistance of heparinase. This review will pave the way to design another sensing probe towards other sulfated contaminants, like dermatan sulfate.
\end{abstract}

Keywords: Colorimetric, Fluorescence, Heparin, Over sulfated chondroitin sulfate, Sensor

\section{Introduction}

$\mathrm{H}$ eparin is biologically recognized as a unique polysaccharide with a large number of sulfated groups, and it is broadly recommended as an anticoagulant drug. After its discovery in 1916 by a medical student of Johns Hopkins University, the structure of heparin was identified to be a building block based on sugar-containing larger molecules with different units of combinations such as uronic acid glucosamine and covalently linked sulfate [1]. These combined units appear to be simple and make heparin one of the strongest acids of nature. Heparin is a polydisperse mixture of sulfonated linear polysaccharides consisting of $75-95 \%$ trisulfated disaccharide repeating unit with molecular weight from 5000 to over 40,000 Da [2]. Later in 1935, a sufficient amount of pure heparin was launched for clinical testing to investigate its treatment efficiency in preventing postoperative thrombosis. Inexpensive and well-tolerated heparin-related therapy efficiently controls thrombosis during surgery and anticoagulant therapy and suppresses the formation of clotting disorders. From then to now, numerous investigators have been devoted to establishing different methods for monitoring plasma heparin since its overdose could lead to detrimental effects, for example, thrombocytopenia and hemorrhaging; the therapeutic levels of heparin are suggested to be 1.7-10 $\mu \mathrm{M}$ for long-term care and $17-67 \mu \mathrm{M}$ for cardiovascular surgery. Classical approaches for quantifying plasma heparin include the activated partial thromboplastin time (aPTT) the prothrombin time test [3]. Unfortunately, the abovediscussed approaches have the limitations of inaccurate measurements due to poor specificity. A

Received 17 June 2021; revised 29 July 2021; accepted 4 August 2021.

Available online 15 December 2021.

* Corresponding author at: Department of Chemistry, National Sun Yat-sen University, No. 70, Lienhai Rd., Kaohsiung, 80424 , Taiwan. Fax: 0118867 3684046.

E-mail address: tsengwl@mail.nsysu.edu.tw (W.-L. Tseng). 
series of review articles have introduced alternative techniques to determine heparin in real-world samples [4,5]. Examples of these techniques include reversed-phase high-performance liquid chromatography (HPLC), anion-exchange chromatography, size-exclusion chromatography (SEC), capillary electrophoresis (CE), polyacrylamide gel electrophoresis (PAGE), nuclear magnetic resonance (NMR) spectroscopy, and mass spectroscopy. Although providing accurate and precise quantification of heparin, these instrumental methods require time-consuming operation and skilled labor. Therefore, developing a portable, sensitive, selective, and fast-response probe is highly needed to detect heparin in clinical samples.

In an effort to satisfy this requirement, the investigators have been devoted to the synthesis of colorimetric and fluorescent sensors toward heparin based on the interplay between the recognition elements and signal reporters [6]. The reported recognition elements encompass coralyne [7,8], surfen [9], AG73 peptides [10], quaternary ammonium moiety-containing molecules [6], protamine [11], and cationic polymers [12]. Moreover, examples of the recently published reporters include chromophores [13], fluorophores [14], polymers [15], metal nanoparticles [16], metal nanoclusters [17], semiconductor quantum dots [18], phosphorescent molecules [19] and metal-organic framework [20]. Since there have been review articles on the fabrication of heparin sensors $[6,21]$, the present review article will focus on summarizing the sensors associated with the detection of a heparinlike glycosaminoglycan, oversulfated chondroitin sulfate (OSCS). The purity and quality of heparin drugs related to the presence of OSCS is an essential issue for the pharmaceutical industry in order to ensure the safety of the heparin supply.
Contaminated heparin could trigger various undesirable side effects that seem to apply to patients, such as angioedema, bleeding complications, hypotension, larynx swelling, and other related symptoms. These symptoms may often have ended up in death. For example, in 2008 , the administration of contaminated heparin caused 149 mortality and 574 reports of adverse reactions in diverse countries [22]. This adverse effect was identified as a result of oversulfated chondroitin sulfate (OSCS), which could be synthesized by sulfonation of low-cost chondroitin sulfate [2]. The mechanism associated with the adverse events of OSCS is mainly involved in the activation of the contact system, triggering the liberation of bradykinin following an allergic reaction [23]. Review articles have extensively covered the advancement of mass spectroscopy-, chromatography-, electrophoresis-, and NMR spectroscopy-related techniques for the determination of OSCS in heparin drug samples [24,25]. These powerful techniques are capable of detecting heparin with the limits of detection (LODs) of OSCS in heparin corresponding to $0.1 \% \mathrm{w} / \mathrm{w}$ in enzyme immunoassay [26], $0.5 \% \mathrm{w} / \mathrm{w}$ in electrochemical detection [27], $\sim 1 \% \mathrm{w} / \mathrm{w}$ in near-infrared (NIR) spectroscopy [28], 0.2\% w/w in PAGE [29], $0.1 \% \mathrm{w} / \mathrm{w}$ in CE [30], and $0.03 \% \mathrm{w} / \mathrm{w}$ in HPLC [31]. However, these published review articles rarely discuss the existing sensors for rapid analysis of OSCS in heparin drug samples. Therefore, the present review article will highlight the recent advances in the fabrication and applications of colorimetric and fluorescent sensors toward OSCS in heparin drug samples. Table 1 summarizes the recently reported probes for detecting OSCS and compares them in terms of linear quantification range, LOD, detection mode and response time.

Table 1. The recently reported probes for detecting OSCS in heparin and compares them in terms of linear range, lowest detectable concentration $(L D C)$, detection mode and analysis time.

\begin{tabular}{|c|c|c|c|c|c|c|c|}
\hline Probe $^{a}$ & Method & Hepari-nase & $\begin{array}{l}\text { Linear range } \\
(\% \mathrm{w} / \mathrm{w})\end{array}$ & $\begin{array}{l}\text { LDC } \\
(\% \mathrm{w} / \mathrm{w})\end{array}$ & $\begin{array}{l}\text { Detection } \\
\text { mode }\end{array}$ & $\begin{array}{l}\text { Analysis } \\
\text { time }\end{array}$ & Ref \\
\hline TPE-conjugated AG73 peptide & FL & Yes & 1 to 70 & 1 & Turn ON & $4 \mathrm{~h}$ & 33 \\
\hline Coralyne-based MB & FL & No & 0.01 to 20 & 0.01 & Turn ON & $5 \mathrm{~min}$ & 38 \\
\hline $\begin{array}{l}\mathrm{MnO}_{2} \text { nanosheets, } \mathrm{SYBR} \text { Green green, } \\
\text { coraylne-based } \mathrm{MB}, \mathrm{Ca}^{2+} \text { ions }\end{array}$ & FL & No & $10^{-10}$ to 10 & $10^{-10}$ & Turn ON & $>60 \mathrm{~min}$ & 39 \\
\hline $\mathrm{Tb}^{3+}, \mathrm{G}-$ rich ssDNA, AG73 Peptide & RTP & Yes & 0.002 to 0.2 & 0.002 & Turn ON & $80 \mathrm{~min}$ & 42 \\
\hline Pyrene-labeled GSRKR & FL & Yes & 0.0001 to 1 & 0.0001 & Ratio & $60 \mathrm{~min}$ & 43 \\
\hline PDDA-TPE as AIE nanoassemblies & FL & No & NA & 1 & Turn OFF & NA & 49 \\
\hline P4Me-3TOEIM & CA & Yes & NA & 0.003 & Spectral shift & $>30 \mathrm{~min}$ & 52 \\
\hline P4Me-3TOEIM & CA & Yes & NA & 0.01 & Spectral shift & $>30 \mathrm{~min}$ & 53 \\
\hline ScGFP, graphene oxide & FL & Yes & NA & $10^{-6}$ & Turn ON & $>4 \mathrm{~h}$ & 55 \\
\hline fluorophore-modified heparin-capped AuNPs & FL & Yes & NA & $10^{-9}$ & Turn ON & $30 \mathrm{~min}$ & 57 \\
\hline ScGFP, rhodafluor dye-labeled heparin & FL & Yes & NA & 0.001 & FRET & $>4 \mathrm{~h}$ & 58 \\
\hline
\end{tabular}

${ }^{a}$ TPE, tetraphenylethen; G, guanine; MB, molecular beacons; AIE, aggregation-induced emission; ScGFP, superpositively charged green fluorescent protein; FL, fluorescence; CA colorimetric assay; RTP, room temperature phosphorescence; NA, not available. 


\section{Challenges for the sensing of OSCS in heparin}

Based on the experience from the design of heparin sensors, the recognition elements used for heparin are still implemented to integrate with the signal reporter for the sensing of OSCS in heparin. The fabrication of OSCS sensors mainly involves: (1) The electrostatic, hydrogen bonding, and hydrophobic interactions between OSCS and the recognition element; (2) the modification of the recognition unit on the signal reporter or the complex formation of the recognition unit with the signal reporter; (3) OSCS-stimulated response of the fabricated sensors. The primary key to sensing OSCS lies in the selectivity of the recognition element toward OSCS in the presence of heparin. However, OSCS has a very similar chemical structure and molecular weight with heparin (Fig. 1) [30]; the difference between OSCS and heparin is the degree of sulfonation. In other words, the recognition element is incapable of discriminating between OSCS and heparin without any further assistance. To overcome this challenge, the investigators have proposed two strategies to identify OSCS in the presence of heparin (Fig. 2). One efficient way to sense OSCS is to combine heparinase digestion with the proposed sensing system. Without the addition of OSCS, heparinase can digest heparin into shortfragment glycosaminoglycan. By contrast, the presence of OSCS efficiently inhibits the enzymatic activity of heparinase [32], preserving the structure of heparin. Since the digested heparin exhibits weaker interaction with the recognition element than the undigested heparin, the detection of OSCS could be successfully achieved using a heparin-related sensor. The limitations of this strategy include the cost of heparinase and the reaction time between heparinase and heparin. The other strategy is to incorporate $\mathrm{Ca}^{2+}$ ions into the sensing system. Earlier published results have shown that $\mathrm{Ca}^{2+}$ ions can specifically bind to the carboxylate end of the iduronate unit as well as the N-sulfamido and 6sulfate moieties of the glucosamine unit in heparin $[33,34]$. This finding suggests that the site-specific interaction takes place between $\mathrm{Ca}^{2+}$ ions and heparin in an aqueous solution. Additionally, in contrast to heparin, OSCS possesses relatively numerous sulfate moieties. As a result, the proposed sensing system can exhibit excellent selectivity toward OSCS in heparin drug samples with the assistance of $\mathrm{Ca}^{2+}$ ions.

\section{Recognition elements}

The core success of the sensor is mainly determined by selecting suitable recognition elements. The recognition elements used for heparin are similar to those applied for OSCS. The published recognition units that identify OSCS are coralyne, AG73 peptides, positively charged tetraphenylethene (TPE) derivatives, polythiophene polymer, poly-L-lysine, protamine, superpositively charged green fluorescent proteins, and poly (diallyldimethylammonium chloride). The features of these recognition units are suggested to have a positively charged structure and a low signal response for chondroitin sulfate (Chs), hyaluronic
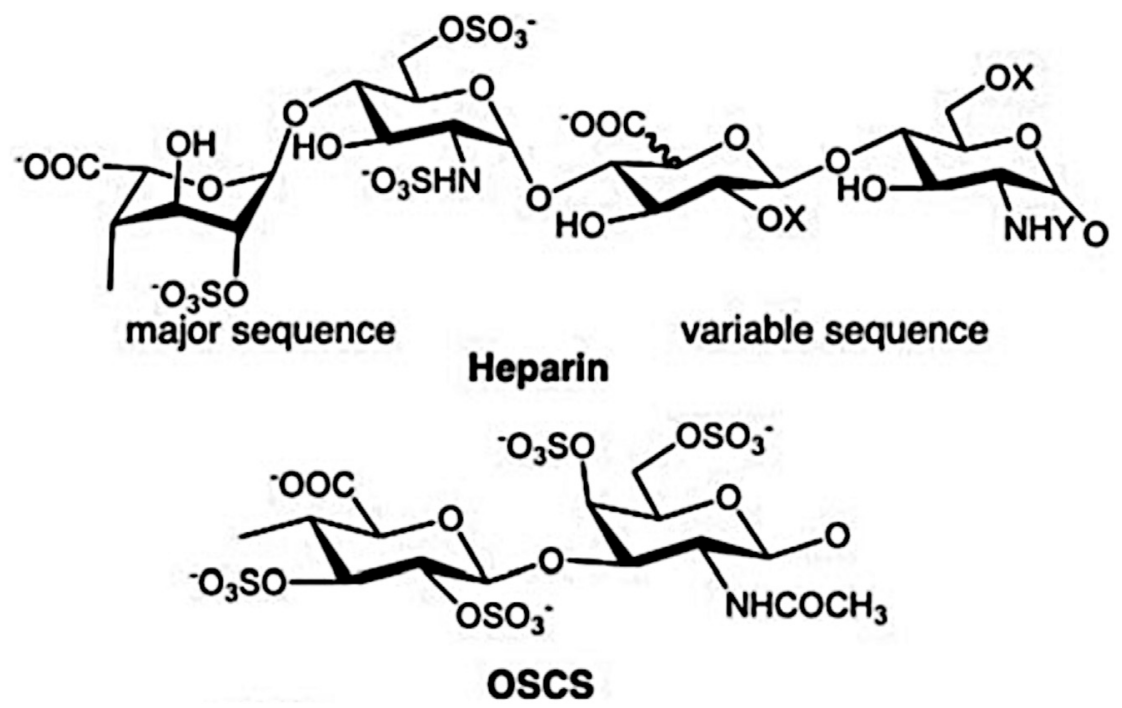

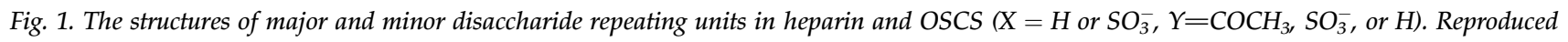
with permission from Ref. [30]. 
(A)

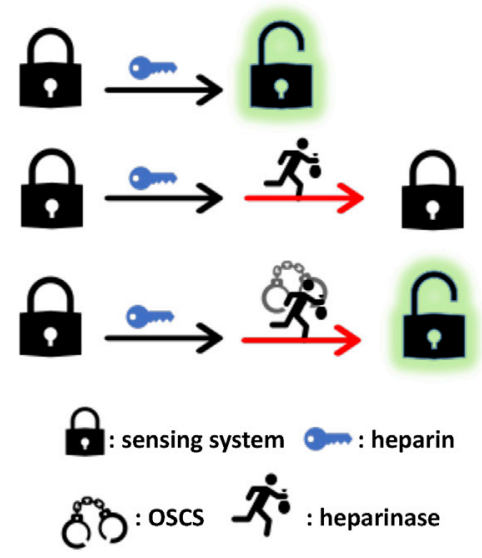

(B)

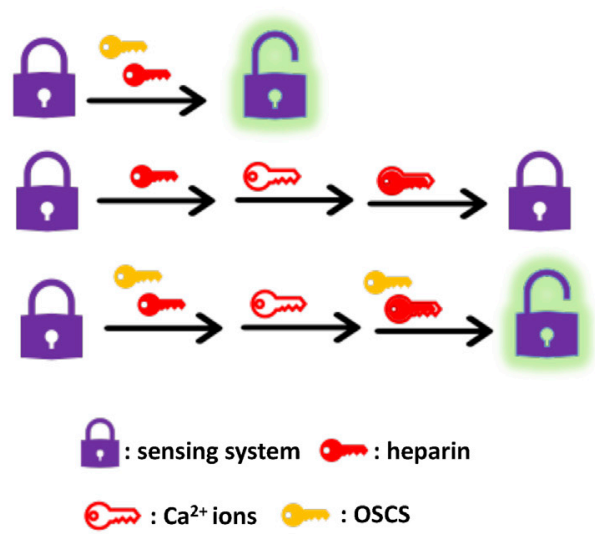

Fig. 2. Strategies for the determination of OSCS in heparin. (A) Integration of heparinase-mediated digestion of heparin and OSCS-induced inhibition of heparinase activity into the sensing system. (B) The addition of $\mathrm{Ca}^{2+}$ ions into the sensing system.

acid (HA), and dextran sulfate (DS). In comparison to other recognition elements, the selectivity of peptide-based system can be tuned by altering the peptide sequence. On the other hand, the selection of the signal reporter could dominate the sensitivity of the sensing system toward OSCS in heparin. Since a non-natural OSCS is a principal contaminant in heparin drug samples, the fluorescent reporters could be well-suited to detect trace OSCS in heparin. We provided a detailed overview of recent literature associated with OSCS sensors based on recognizing recognition units.
(A)<smiles>COc1cc2cc[n+](C)c(C)c2cc1OC</smiles>

(C)

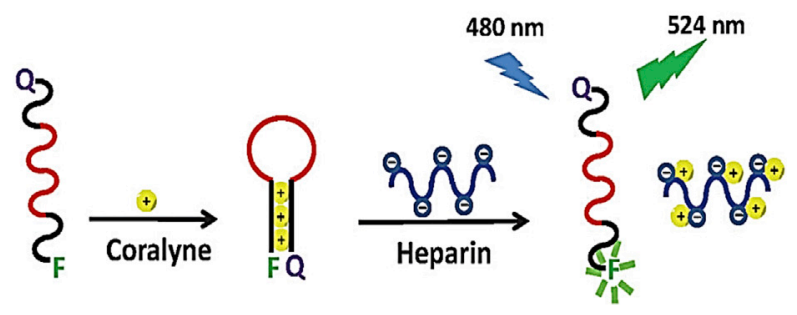

\subsection{Coralyne}

Coralyne possesses a planar structure (Fig. 3A) and specifically binds to polydeoxyadenosine [poly (dA)] through the coordination of four adenine bases with one coralyne. The association constant of coralyne and poly (dA) was reported to be $1.8 \times 10^{6} \mathrm{M}^{-1}$ [35]. Additionally, coralyne exhibits strong electrostatic attraction with negatively charged sulfate glycosaminoglycan, for example, dextran sulfate and chondroitin-6-sulfate [36]. The above-discussed features enable the fabrication of
(B)

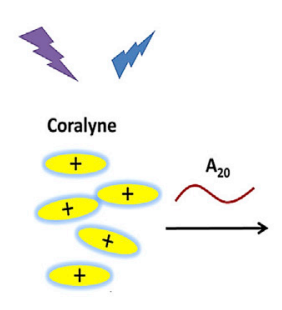

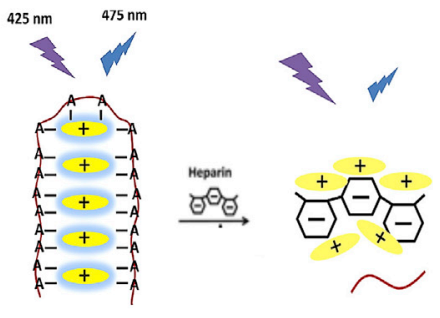

(D)

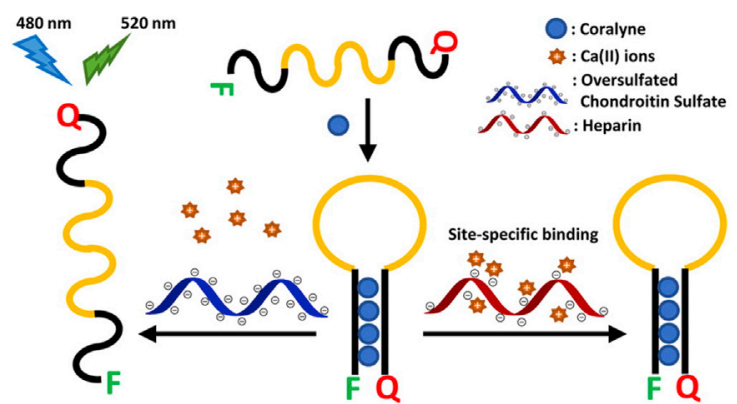

Fig. 3. Schematic illustration of coralyne-based sensors for detecting heparin and OSCS. (A) Chemical structure of coralyne. (B) The $A_{20}-$ coralyne complex for fluorescence turn-off detection of heparin through the competitive binding between $A_{20}$ and heparin for coralyne. Reproduced with permission from Ref. [7]. (C) The coralyne-based MB as a fluorescent turn-on sensor for heparin through the removal of coralyne from the $A_{20}$ stem. Reproduced with permission from Ref. [8]. (D) The coralyne-based MB for sensing OCSCS in heparin based on the use of Ca ${ }^{2+}$ ions as a masking agent for heparin. Reproduced with permission from Ref. [37]. 
the coralyne-polyadenosine (polyA) complex-based sensors to detect heparin via the interplay of coralyne, polyA, and heparin. For example, Hung et al. proposed the fluorescent probe based on the direct mixing of 20-repeat adenosine $\left(\mathrm{A}_{20}\right)$ and coralyne as a complex (Fig. 3B) [7]. The complexed coralyne emitted strong blue fluorescence due to the restriction of its rotation. The presence of heparin was capable of triggering the removal of coralyne from the $\mathrm{A}_{20}$-coralyne complexes. As a result, the fluorescence of coralyne was gradually quenched with increasing the concentration of heparin. Guided by the same concept, $\mathrm{Ku}$ et al. designed an adenosinebased molecular beacon (MB) with a pair of 12-mer adenosine bases in the stem (Fig. 3C) [8]. The MB was labeled with a quencher at the $3^{\prime}$-end and a fluorescent reporter at the $5^{\prime}$-end. Upon the addition of coralyne interacting with 12-mer adenosine bases, the structural conformation of the MB was converted from a random coil to a hairpin. The conformation change of the MB brought the closeness of a quencher to a fluorophore. By learning from previous experience in the design of coralynebased sensors toward heparin, Lee et al. found that the presence of $\mathrm{Ca}^{2+}$ ions allowed an adenosinebased MB to probe $0.01 \% \mathrm{w} / \mathrm{w}$ OSCS in the pharmaceutical heparin within 5 min (Fig. 3D) [37]. The proposed $\mathrm{MB}$ still kept excellent selectivity toward OSCS when substituting $\mathrm{CaCl}_{2}$ with $\mathrm{CaCO}_{3}$ or $\mathrm{Ca}\left(\mathrm{NO}_{3}\right)_{2}$, providing clear evidence associated with the interaction between $\mathrm{Ca}^{2+}$ ions with heparin. Similarly, Tian et al. reported the combination of (i) a hybridization chain reaction of two MBs, (ii) a coralyne-containing $\mathrm{MB}$ that is responsive to heparin and OSCS, (iii) SYBR Green I-adsorbed $\mathrm{MnO}_{2}$ nanosheets, (iv) double stranded-mediated fluorescence enhancement of SYBR Green I, and (v) the use of $\mathrm{Ca}^{2+}$ ions as an additive for the construction of the sensing system for OSCS. The proposed sensing system can detect $10^{-8} \% \mathrm{w} / \mathrm{w}$ OSCS in the presence of heparin [38].

\subsection{Peptide}

A previous study reported a specific interaction between an AG73 peptide and heparin with a dissociation constant of $6.4 \mathrm{mM}$ [39]. The amino acid sequence of an AG73 peptide has been determined to be RKRLQVQLSIRT with an isoelectric point of 12.4. Such kind of peptide owns the $\mathrm{pK}_{\mathrm{a}}$ values of 12.4 and 10.5 for guanidyl group in an arginine residue and $\mathrm{NH}_{3}^{+}$group in a lysine residue, respectively. These positively charged groups enable an AG73 peptide to have a specific interaction with glycosaminoglycan-expressed cells. Thus, the probe consisting of an AG73 peptide and a reported element is commonly designed to detect heparin without the interferences of other sulfated glycosaminoglycans. As an example, You and Tseng found that an AG73 peptide was capable of triggering the aggregation of glutathione-capped gold nanoclusters, switching on their luminescence (Fig. 4A) [10]. The appearance of heparin quenched the luminescence of the above-discussed aggregates through the specific interaction between heparin and AG73 peptides. The LOD of heparin, detected by this strategy, was determined to be $3 \mathrm{nM}$. Cheng et al. prepared iron-porphyrin derivative-related metal-organic framework (MOF) nanosheets as a peroxidase-like nanoenzyme and modified them with AG73 peptides through physical adsorption [40]. The resultant AG73-modified MOF nanosheets exhibited low catalytic activity for $\mathrm{H}_{2} \mathrm{O}_{2}$-mediated oxidation of Ampliflu Red since AG73 peptides blocked the active sites. The presence of heparin restored the catalytic activity of AG73 peptidemodified MOF nanosheets via the liberation of AG73 peptides from the MOF nanosheet surface. The above-discussed probe provided the LOD of heparin corresponding to $15 \mathrm{ng} / \mathrm{mL}$ with a low response to $\mathrm{Chs}$ and $\mathrm{HA}$. Considering that the chemical properties of OSCS resemble those of heparin, the investigators have proposed that an AG73 peptide also has the similar interaction with OSCS. Ding et al. developed the AG73 peptideconjugated tetraphenylethene probe and showed a similar fluorescence turn-on response to heparin and OSCS [32] (Fig. 4B). The introduction of heparin and OSCS both triggered the aggregation of the positively charged probe, lighting up the fluorescence of tetraphenylethene resides. This phenomenon is identified as aggregation-induced emission (AIE). Once present in the sensing system, heparinase induced the cleavage of heparin. As a result, the hydrolyzed heparin rarely promoted the aggregation of the AG73 peptide-conjugated probe without the enhanced fluorescence of tetraphenylethene resides. Given that OSCS can inhibit the enzymatic activity of heparinase, a mixture of OSCS and heparin still switched on the fluorescence of the AG73 peptide-conjugated probe in the presence of heparinase. Thus, the system mentioned above could sense a trace amount of OSCS in heparin with the LOD of $0.001 \% \mathrm{w} / \mathrm{w}$. Hu et al. reported a timeresolved luminescence (TRL) system for sensitive and selective detection of OSCS in heparin with the assistant of heparinase (Fig. 4C) [41]. The sensing procedure can be divided into four steps: (i) guanine-rich single-stranded DNA (ssDNA) molecules complex with AG73 peptides through electrostatic 
(A)

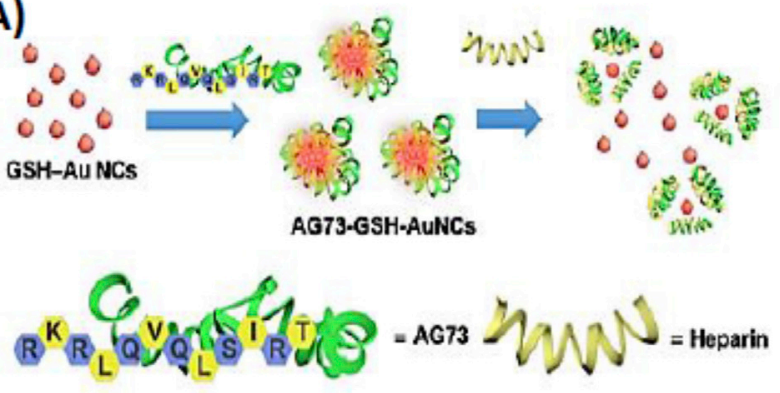

(c)
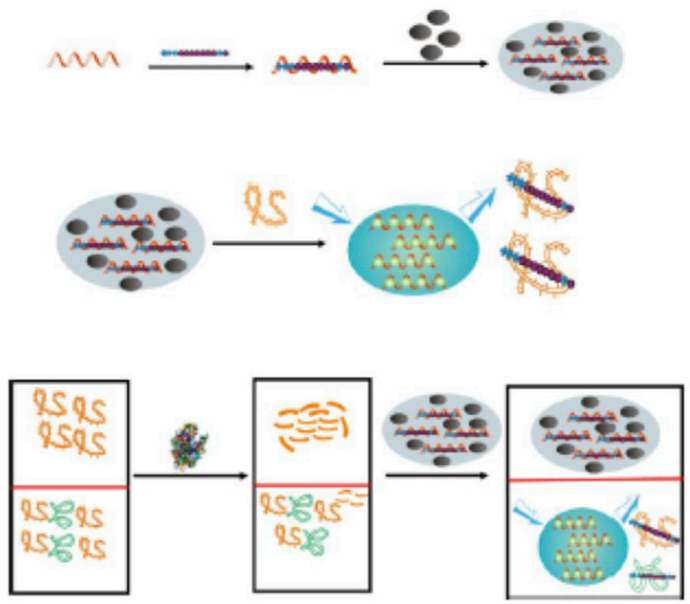

(B)

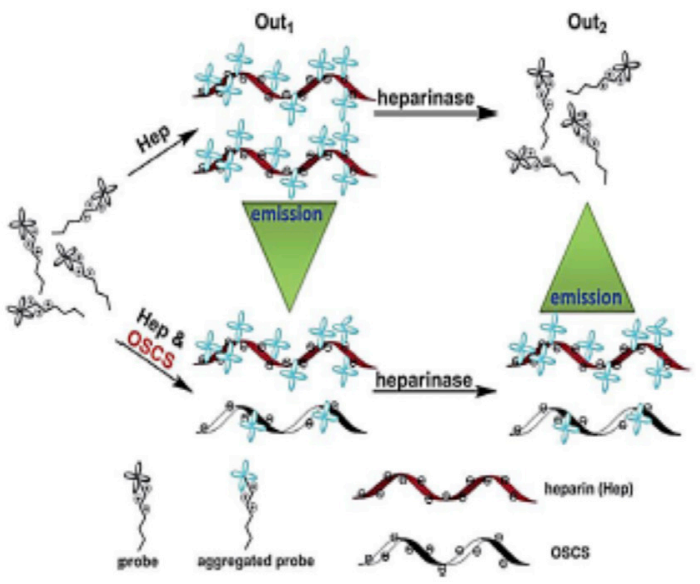

(D)

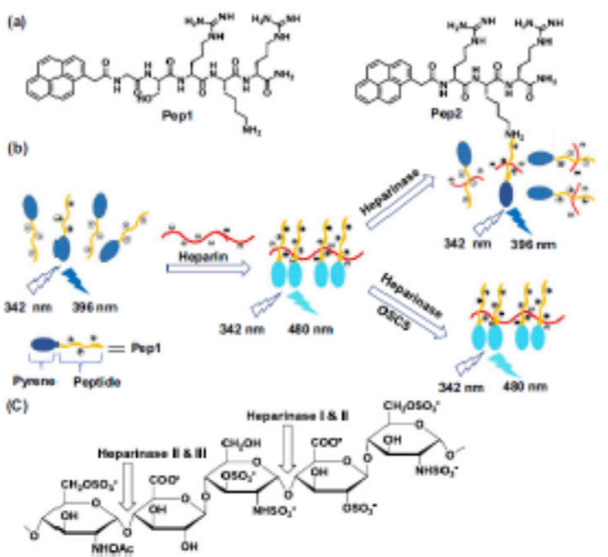

Fig. 4. Schematic illustration of peptide-related sensors for detecting OSCS and heparin. (A) The nanocomposites of AG73 peptides and glutathionecapped gold nanoclusters as a fluorescent turn-off probe for heparin via the specific interaction of heparin and AG73 peptides. Reproduced with permission from Ref. [10]. (B) Fluorescent sensing of OSCS in heparin based on the combination of heparin- and OSCS-induced AIE of AG73 peptide-modified tetraphenylethene and heparinase-mediated hydrolysis of heparin. Reproduced with permission from Ref. [32]. (C) Fabrication of the sensing system toward OSCS by the complexation of $s s D N A$ and $T b^{3+}$, electrostatic attraction of ssDNA with AG73 peptides, specific interaction of AG73 peptides with OSCS and heparin, and heparinase-mediated digestion of heparin. Reproduced with permission from Ref. [41]. (D) Design of a fluorescent probe for the detection of OSCS in heparin by heparin-triggered AIE of GSRKR-conjugated pyrene and OSCS-mediated inhibition of heparinase activity. Reproduced with permission from Ref. [42].

attraction; (ii) $\mathrm{Tb}^{3+}$ ions are introduced into the formed complexes; (iii) the presence of heparin or OSCS triggers the release of AG73 peptides from the complexes, allowing $\mathrm{Tb}^{3+}$ ions to interact with guanine-rich ssDNA molecules; (iv) the formed luminescence complexes exhibit strong green emission since guanine bases behave as an effective antenna ligand. After heparinase catalyzed the cleavage of the heparin's saccharide bonds, the hydrolyzed heparin was incapable of activating the release of AG73 peptides from the complexes. In other words, the TRL system was insensitive to heparin in the presence of heparinase. By contrast, heparinase exhibited low catalytic activity toward OSCS. Thus, upon the addition of heparinase, the TRL system was still highly responsive to OSCS, allowing the detectable concentration as low as $0.002 \% \mathrm{w} / \mathrm{w}$ of OSCS in heparin. In addition to
AG73 peptides, Mehta et al. designed a heparinresponsive peptide (amino acid sequence of GSRKR) and modified its tail group with a pyrene (Fig. 4D) [42]. The arginine units of the proposed pyrene-modified peptide were recognized as a binding site for heparin. Under an excitation wavelength of $342 \mathrm{~nm}$, the pyrene fluorophore emitted fluorescence at $396 \mathrm{~nm}$ and $480 \mathrm{~nm}$ in the monomer and excimer states. As heparin and OSCS were added, the as-made peptides were assembled onto their backbone via electrostatic attraction. Consequently, the aggregated peptides facilitated the conjugated pyrene fluorophores to approach each other, leading to a redshift in their maximum emission wavelength from 396 to $480 \mathrm{~nm}$. These features allowed the proposed pyrene-modified peptide to sense heparin and OSCS in an aqueous solution ratiometrically. The above-discussed probe 
integrated with heparinase-mediated hydrolysis of heparin was powerful to detect $0.0001 \% \mathrm{w} / \mathrm{w}$ OSCS in heparin.

\subsection{Poly(diallyldimethylammonium) chloride (PDDA)}

A cationic PDDA is well-known to apply various areas, such as an additive in capillary electrophoresis [43], a polycation in a layer-by-layer technique [44], and an exfoliating agent in the preparation of monolayer two-dimensional nanomaterials [45]. Moreover, PDDA can serve as a platform for electrostatic attraction and $\pi-\pi$ interactions with negatively charged polyelectrolyte and nanomaterials [46]. In light of these features, Yang et al. disclosed that PDDA induced the assembly of negatively charged benzoperylene derivatives (BPD) through multiple interactions, leading to the conversion of BPD from monomers to excimers [12]. Due to the apparent difference in maximum emission wavelength of BPD between monomers and excimers, the complexes of PDDA and BPD excimers were implemented for ratiometric sensing of heparin through the heparin-mediated liberation of PDDA from the complexes. The as-made complexes provided accurate and precise quantification of $0.4-3.5 \mu \mathrm{M}$ heparin with the LOD of $50 \mathrm{nM}$. However, $5 \mu \mathrm{M}$ Chs could have a higher ratiometric response than $1 \mu \mathrm{M}$ heparin. Likewise, Qiao et al. exploited PDDA to activate the aggregation of sulfonate-ended pyrene, converting their monomers to excimers [47]. The formed complexes of PDDA and pyrene derivative excimers were shown to serve as a fluorescent ratiometric probe for quantifying 0.1-2.3 $\mu \mathrm{M}$ heparin through the restoration of excimers to monomers. The PDDA-pyrene derivative complexes were highly sensitive to heparin without the interferences of $\mathrm{Chs}$ and HA. Since complexing with heparin, PDDA polymers are expected to have the same interaction strength with OSCS. By assembling a PDDA polymer with carboxyl group-terminated tetraphenylethene (TPE) molecules, Yang et al. fabricated an AIE-based sensor to detect heparin and OSCS [48]. The aggregation degree of TPE molecules complexed with a PDDA polymer can be tuned by simply adjusting the mixed ratio of TPE to PDDA. The TPE-PDDA assemblies emitted strong fluorescence at $466 \mathrm{~nm}$, whereas the added heparin promoted their fluorescence quenching due to the formation of the PDDA-heparin complexes. A similar result was observed as OSCS was used in place of heparin. The sensitivity of the AIE sensor toward heparin and OSCS was highly connected with the aggregation degree of TPE molecules in the assemblies. The TPE-PDDA assemblies were substantiated to be useful in detecting a trace amount of OSCS contamination in heparin.

\subsection{Cationic polythiophene derivatives}

Leclerc's group pioneered the synthesis of poly (1methyl-3-[2-[(4-methyl-3-thienyl)oxy]ethyl]-1H-imidazolium) [P4Me-3TOEIM] based on the oxidative polymerization [49]. The chemical structures of P4Me-3TOEIM consisting of 4-methyl residue on each thiophene unit. In the absence of negatively charged oligomers and polymers, P4Me-3TOEIM adopts a random coil structure in an aqueous solution, forcing its conjugated backbone to twist in an aqueous solution and to reduce the effective conjugation length. By contrast, the introduction of negatively charged oligomers and polymers can cause the conformation change of P4Me-3TOEIM from a random coil structure to a double helix-like strand. As an outcome, the extended conjugation length of P4Me-3TOEIM causes a red-shift in its absorption band. The characteristics mentioned above inspired the investigators to propose the utilization of P4Me-3TOEIM for the sensing of heparin through electrostatic attraction. Zhan et al. employed P4Me-3TOEIM as a colorimetric sensor for the naked-eye detection of heparin in bovine serum [50]. The presence of heparin induced the conformation alternation of P4Me-3TOEIM, and as a consequence, its maximum absorption wavelength shifted to a higher value. In other words, the absorption intensity of P4Me-3TOEIM at $500 \mathrm{~nm}$ was gradually enhanced with increasing the heparin concentration. The LODs of heparin in water and bovine serum obtained from P4Me-3TOEIM were reported to be 0.01 and $0.15 \mathrm{U} / \mathrm{mL}$, respectively. Sommers and Keire took the advantages of P4Me3TOEIM to design a convenient probe for the naked-eye sensing of OSCS in heparin based on (i) OSCS-mediated inhibition of heparinase I and II activity and (ii) the digested heparin-induced color change of P4Me-3TOEIM [51]. It was found that P4Me-3TOEIM exhibited a higher colorimetric response to dermatan sulfate and Chs (partially sulfated form) than OSCS and heparin (oversulfated form). Accordingly, the selectivity of P4Me-3TOEIM could be dominated by the tertiary structure of sulfated glycosaminoglycans rather than their sulfation degree. As OSCS inhibited heparinase activity, the undigested heparin only caused a slight color change of P4Me-3TOEIM. The P4Me-3TOEIM probe successfully detected $0.003 \% \mathrm{w} / \mathrm{w}$ in heparin sodium active pharmaceutical ingredient and $0.1 \%$ 
$\mathrm{w} / \mathrm{w}$ in the crude heparin samples. The same group disclosed that the absorption band of P4Me3TOEIM is susceptible to the molecular weight of heparin [52]. The P4Me-3TOEIM probe, relying on the same sensing mechanism, was employed to identify $0.01 \% \mathrm{w} / \mathrm{w}$ OSCS in commercially available low-molecular-weight heparin (M.W. ca. 4000-6000), such as enoxaparin, dalteparin, and tinzaparin. Dermatan sulfate and Chs have been recognized as contaminants in heparin drug samples. Toby et al. integrated the P4Me-3TOEIM probe with chondroitinase digestion and centrifugal filtration to analyze dermatan sulfate and Chs in heparin [53]. The role of chondroitinase is to cleavage chondroitins, while the functionality of centrifugal filtration is to separate a mixture of digested chondroitins and heparin. This sensing system was feasible for monitoring $0.5 \% \mathrm{w} / \mathrm{w}$ dermatan sulfate and $1.0 \% \mathrm{w} / \mathrm{w}$ Chis in heparin.

\subsection{Fluorescent proteins}

Superpositively charged green fluorescent proteins (ScGFPs) were introduced for the sensing of heparin due to their distinct advantages of satisfactory biocompatibility, good water solubility, and numerous positively charged groups (a net charge of +36 ). It is expected that ScGFPs could strongly bind to heparin via electrostatic attraction and hydrophobic interaction. According to these features, Wang et al. developed a fluorescence turn-on probe for selective detection of OSCS in heparinasetreated heparin [54]. The sensing mechanism mainly involved the following concepts (Fig. 5A): (i) superpostively charged ScGFPs were electrostatically assembled on the surface of graphene oxide, quenching their fluorescence based on energy or charge transfer; (ii) superpostively charged ScGFPs electrostatically interact with heparin without the interference of their fluorescence; (iii) the complexes of ScGFPs and heparin were rarely adsorbed on the surface of graphene oxide due to electrostatic repulsion between them; (iv) OSCS inhibited the digestion of heparin by heparinase. By the interplay among of ScGFPs, graphene oxides, and heparinase, the sensing system was capable of quantifying 3.3-66.7 $\mathrm{nM}$ heparin in $10 \%$ human plasma and $10^{-9}$ to $10 \% \mathrm{w} / \mathrm{w}$ OSCS in heparin.

(A)

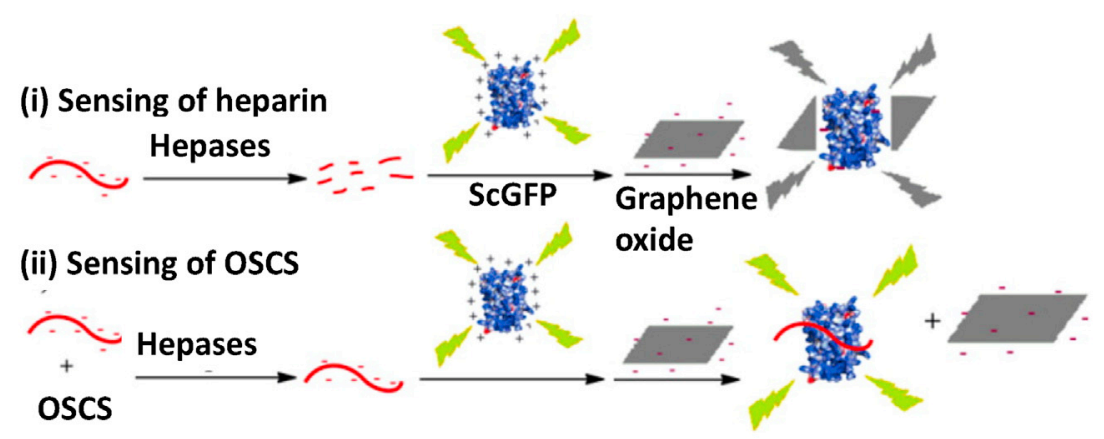

Hepases: Heparinase

(B)

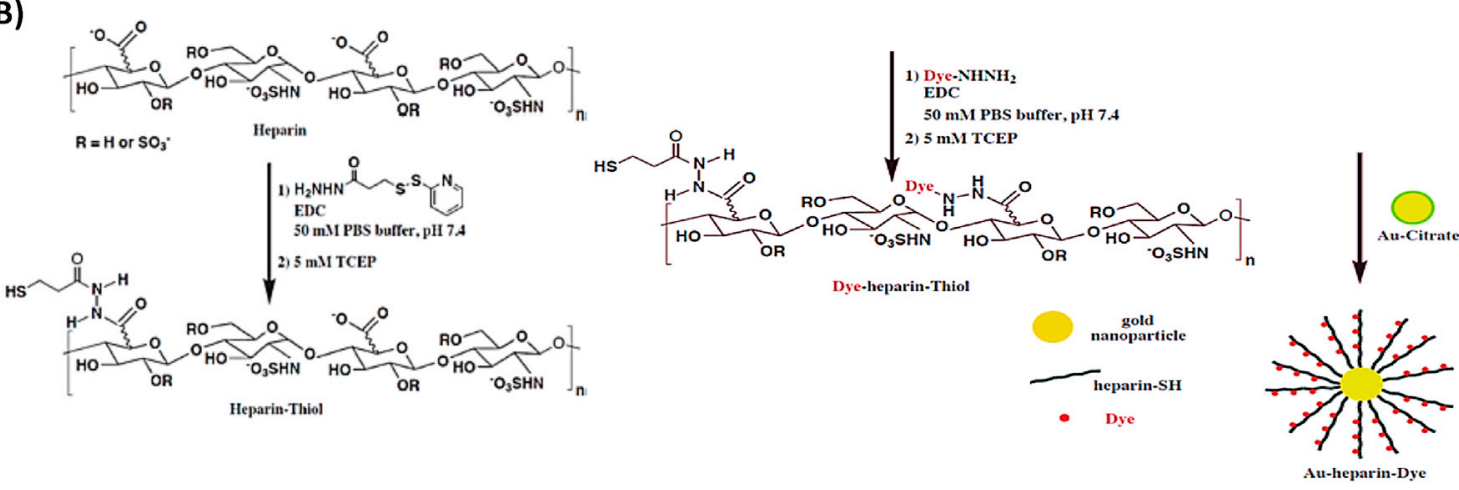

Fig. 5. (A) Fluorescent sensing of OSCS in heparin based on graphene oxide-induced fluorescence quenching of ScGFP, heparin-promoted removal of ScGFP from graphene oxide, and OSCS-mediated suppression of heparinase activity. Reproduced with permission from Ref. [54] (B) Modification of heparin with thiol groups and fluorophores, followed by the conjugation to the surface of citrate-capped AuNPs. Reproduced with permission from Ref. [56]. 


\subsection{Organic dye-labeled heparin}

It is well-documented that heparinase specifically cleaves heparin, and its activity is inhibited by OSCS [32,55]. According to this information, Kalita et al. modified heparin with thiol groups and HiLyte Fluor 594 fluorophores, resulting in thiol- and HiLyte Fluor production 594-conjugated heparin [56]. Subsequently, the thiol- and HiLyte Fluor 594conjugated heparin was directly attached to the surface of citrate-capped gold nanoparticles (AuNPs) through the formation of $\mathrm{Au}-\mathrm{S}$ bonds (Fig. 5B). The fluorescence of the conjugated HiLyte Fluor 594 in heparin was efficiently quenched by the AuNPs as a result of nanometal surface energy transfer. The presence of heparinase digested the adsorbed heparin, leading to the release of HiLyte Fluor 594 from the nanoparticle surface. By contrast, the added OSCS blocked heparinase activity, suppressing the liberation of HiLyte Fluor 594. Taken together, the fluorescence of the conjugated HiLyte Fluor 594 was progressively reduced with increasing the concentration of OSCS in heparin. This sensing platform identified $10^{-9} \% \mathrm{w} / \mathrm{w}$ OSCS in heparin within $30 \mathrm{~min}$. Additionally, Ding et al. fabricated the complexes of ScGFPs and rhodafluor dyelabeled heparin [57]. The fluorescence resonance energy transfer (FRET) occurred from ScGFPs to rhodafluor dye-labeled heparin in the formed complexes due to the spectral overlap between the emission of ScGFPs and the absorption of rhodafluor dye. Heparinase was introduced to cleavage rhodafluor dye-labeled heparin in the formed complexes, producing free rhodafluor dye-labeled heparin fragments without complexing with ScGFPs. In other words, a FRET efficiency between ScGFPs and rhodafluor dye-labeled heparin was inversely dependent on the level of heparinase; however, OSCS blocked the phenomenon mentioned above. The combination of heparinase and the as-made complexes was well-suited for ratiometric sensing of $0.001 \% \mathrm{w} / \mathrm{w}$ OSCS in heparin.

\section{Conclusion and future trends}

Most of the probes mentioned above are highly involved in heparinase treatment owing to the high specificity of heparinase toward heparin and OSCSinduced inhibition of its activity. However, this strategy could have some limitations: (i) heparinase activity is sensitive to environmental variations, such as $\mathrm{pH}$ and temperature; (ii) heparinase takes a long time to digest heparin ( $>1 \mathrm{~h}$ ); (iii) expensive heparinase requires temperature and humidity controls; (iv) the prolong incubation of OSCS with heparinase is needed to block its activity. Hence, we recommend that the challenge in this field will be to develop the probe for rapid sensing of OSCS in the absence of heparinase. On the other hand, the most common contaminant in heparin is known as dermatan sulfate. The level of dermatan sulfate is determined to be $1-7 \%$ in heparin. Since dermatan sulfate rarely affects the anticoagulation outcome of heparin, the investigator barely focuses on developing the sensor toward dermatan sulfate. However, the United States Pharmacopoeia (USP) recommended that the level of dermatan sulfate impurities should be lower than $1 \%$ in heparin. The building blocks of dermatan sulfate comprise four possible hexosamines and three possible uronic acids. Dermatan sulfate contains specific iduronic acid, which is effective in distinguishing it from heparan sulfate and heparin. However, unlike OSCS and heparin, dermatan sulfate holds a relatively low negative charge. Accordingly, it is another challenge to rationally design a suitable probe for dermatan sulfate without heparin interference.

\section{Conflict of interest}

The authors have declared no conflict of interest.

\section{Acknowledgment}

This work was financially supported by the Ministry of Science and Technology of Taiwan, Taiwan under contract number MOST 107-2113-M-110 -013 -MY3.

\section{References}

[1] Capila I, Linhardt RJ. Heparin-protein interactions. Angew Chem Int Ed 2002;41:390-412.

[2] Liu H, Zhang Z, Linhardt RJ. Lessons learned from the contamination of heparin. Nat Prod Rep 2009;26:313-21.

[3] Girolami B, Girolami A. Heparin-induced thrombocytopenia: a review. In: Seminars in thrombosis and hemostasis Citeseer; 2006. p. 803-9.

[4] Yang B, Solakyildirim K, Chang Y, Linhardt RJ. Hyphenated techniques for the analysis of heparin and heparan sulfate. Anal Bioanal Chem 2011;399:541-57.

[5] Qiao M, Lin L, Xia K, Li J, Zhang X, Linhardt RJ. Recent advances in biotechnology for heparin and heparan sulfate analysis. Talanta 2020;219:121270.

[6] Fan L, Jia D, Zhang W, Ding Y. Chemical sensors for selective and quantitative heparin sensing. Analyst 2020;149: 7809-24.

[7] Hung S-Y, Tseng W-L. A polyadenosine-coralyne complex as a novel fluorescent probe for the sensitive and selective detection of heparin in plasma. Biosens Bioelectron 2014;57: 186-91.

[8] Kuo C-Y, Tseng W-L. Adenosine-based molecular beacons as light-up probes for sensing heparin in plasma. Chem Commun 2013;49:4607-9.

[9] You J-G, Wang Y-T, Tseng W-L. Adenosine-related compounds as an enhancer for peroxidase-mimicking activity of nanomaterials: application to sensing of heparin level in 
human plasma and total sulfate glycosaminoglycan content in synthetic cerebrospinal fluid. ACS Appl Mater Interfaces 2018;10:37846-54.

[10] You J-G, Tseng W-L. Peptide-induced aggregation of glutathione-capped gold nanoclusters: a new strategy for designing aggregation-induced enhanced emission probes. Anal Chim Acta 2019;1078:101-11.

[11] Aparna R, Devi JA, Anjana R, Nebu J, George S. Reversible fluorescence modulation of BSA stabilised copper nanoclusters for the selective detection of protamine and heparin. Analyst 2019;144:1799-808.

[12] Yang M, Chen J, Zhou H, Li W, Wang Y, Li J, et al. Polycation-induced benzoperylene probe excimer formation and the ratiometric detection of heparin and heparinase. Biosens Bioelectron 2016;75:404-10.

[13] Bhaumik SK, Banerjee S. Highly sensitive and ratiometric luminescence sensing of heparin through templated cyanostilbene assemblies. Analyst 2021;146:2194-202.

[14] Jana P, Radhakrishna M, Khatua S, Kanvah S. A "turn-off" red-emitting fluorophore for nanomolar detection of heparin. Phys Chem Chem Phys 2018;20:13263-70.

[15] Pu K-Y, Liu B. A multicolor cationic conjugated polymer for naked-eye detection and quantification of heparin. Macromolecules 2008;41:6636-40.

[16] You J-G, Liu Y-W, Lu C-Y, Tseng W-L, Yu C-J. Colorimetric assay of heparin in plasma based on the inhibition of oxidase-like activity of citrate-capped platinum nanoparticles. Biosens Bioelectron 2017;92:442-8.

[17] Hu L, Liao H, Feng L, Wang M, Fu W. Accelerating the peroxidase-like activity of gold nanoclusters at neutral $\mathrm{pH}$ for colorimetric detection of heparin and heparinase activity. Anal Chem 2018;90:6247-52.

[18] Peng X, Long Q, Li H, Zhang Y, Yao S. "Turn on-off" fluorescent sensor for protamine and heparin based on label-free silicon quantum dots coupled with gold nanoparticles. Sensor Actuator B Chem 2015;213:131-8.

[19] Jiang J, Zhang C, Lin W, Liu Y, Liu S, Xu Y, et al. Long-lived phosphorescent Iridium (iii) complexes conjugated with cationic polyfluorenes for heparin sensing and cellular imaging. Macromol Rapid Commun 2015;36:640-6.

[20] Liu L, Dai J, Ji Y, Shen B, Zhang X, Linhardt RJ. Detection of protamine and heparin using a promising metal organic frameworks based fluorescent molecular device BZA-BOD@ ZIF-90. Sensor Actuator B Chem 2021;341:130006.

[21] Bromfield SM, Wilde E, Smith DK. Heparin sensing and binding-taking supramolecular chemistry towards clinical applications. Chem Soc Rev 2013;42:9184-95.

[22] McMahon AW, Pratt RG, Hammad TA, Kozlowski S, Zhou E, $\mathrm{Lu} \mathrm{S}$, et al. Description of hypersensitivity adverse events following administration of heparin that was potentially contaminated with oversulfated chondroitin sulfate in early 2008. Pharmacoepidemiol Drug Saf 2010;19:921-33.

[23] Adam A, Montpas N, Keire D, Désormeaux A, Brown NJ, Marceau F, et al. Bradykinin forming capacity of oversulfated chondroitin sulfate contaminated heparin in vitro. Biomaterials 2010;31:5741-8.

[24] Beni S, Limtiaco JF, Larive CK. Analysis and characterization of heparin impurities. Anal Bioanal Chem 2011;399:527-39.

[25] Ramacciotti E, Clark M, Sadeghi N, Hoppensteadt D, Thethi I, Gomes M, et al. Contaminants in heparin: review of the literature, molecular profiling, and clinical implications. Clin Appl Thromb Hemost 2011;17:126-35.

[26] Bairstow S, McKee J, Nordhaus M, Johnson R. Identification of a simple and sensitive microplate method for the detection of oversulfated chondroitin sulfate in heparin products. Anal Biochem 2009;388:317-21.

[27] Wang L, Buchanan S, Meyerhoff ME. Detection of highcharge density polyanion contaminants in biomedical heparin preparations using potentiometric polyanion sensors. Anal Chem 2008;80:9845-7.

[28] Spencer JA, Kauffman JF, Reepmeyer JC, Gryniewicz CM, Ye W, Toler DY, et al. Screening of heparin API by near infrared reflectance and Raman spectroscopy. J Pharmacol Sci 2009:98:3540-7.

[29] Zhang Z, Li B, Suwan J, Zhang F, Wang Z, Liu H, et al. Analysis of pharmaceutical heparins and potential contaminants using 1H-NMR and PAGE. J Pharmaceut Sci 2009;98: 4017-26.

[30] Volpi N, Maccari F, Suwan J, Linhardt RJ. Electrophoresis for the analysis of heparin purity and quality. Electrophoresis 2012;33:1531-7.

[31] Trehy ML, Reepmeyer JC, Kolinski RE, Westenberger BJ, Buhse LF. Analysis of heparin sodium by SAX/HPLC for contaminants and impurities. J Pharmaceut Biomed Anal 2009;49:670-3.

[32] Ding Y, Shi L, Wei H. A "turn on" fluorescent probe for heparin and its oversulfated chondroitin sulfate contaminant. Chem Sci 2015;6:6361-6.

[33] Rabenstein DL, Robert JM, Peng J. Multinuclear magnetic resonance studies of the interaction of inorganic cations with heparin. Carbohydr Res 1995;278:239-56.

[34] Chevalier F, Lucas R, Angulo J, Martin-Lomas M, Nieto PM. The heparin-Ca2+ interaction: the influence of the O-sulfation pattern on binding. Carbohydr Res 2004;339:975-83.

[35] Persil O, Santai CT, Jain SS, Hud NV. Assembly of an antiparallel homo-adenine DNA duplex by small-molecule binding. J Am Chem Soc 2004;126:8644-5.

[36] Megyesi M, Biczók L, Görner HJP. Dimer-promoted fluorescence quenching of coralyne by binding to anionic polysaccharides. Photochem Photobiol Sci 2009;8:556-61.

[37] Lee C-Y, Tseng W-L. Molecular beacon-based fluorescent assay for specific detection of oversulfated chondroitin sulfate contaminants in heparin without enzyme treatment. Anal Chem 2015;87:5031-5.

[38] Tian R, Jiang H, Wang G. MnO 2 nanosheet-based heparin and OSCS fluorescent biosensor with lowered background and amplified hybridization chain reaction. RSC Adv 2016;6: 89803-9.

[39] Hoffman MP, Engbring JA, Nielsen PK, Vargas J, Steinberg Z, Karmand AJ, et al. Cell type-specific differences in glycosaminoglycans modulate the biological activity of a heparin-binding peptide (RKRLQVQLSIRT) from the G domain of the laminin $\alpha 1$ chain. J Biol Chem 2001;276: 22077-85.

[40] Cheng H, Liu Y, Hu Y, Ding Y, Lin S, Cao W, et al. Monitoring of heparin activity in live rats using metal-organic framework nanosheets as peroxidase mimics. Anal Chem 2017;89:11552-9.

[41] Hu Y, Guo W, Ding Y, Cheng H, Wei H. Modulating luminescence of $\mathrm{Tb} 3+$ with biomolecules for sensing heparin and its contaminant OSCS. Biosens Bioelectron 2016;86:858-63.

[42] Mehta PK, Lee H, Lee K-H. Highly sensitive ratiometric detection of heparin and its oversulfated chondroitin sulfate contaminant by fluorescent peptidyl probe. Biosens Bioelectron 2017;91:545-52.

[43] Yeh P-R, Tseng W-L. Human serum albumin-coated gold nanoparticles for selective extraction of lysozyme from realworld samples prior to capillary electrophoresis. J Chromatogr A 2012;1268:166-72.

[44] Lin YW, Tseng WL, Chang HT. Using a layer-by-layer assembly technique to fabricate multicolored-light-emitting films of CdSe@ CdS and CdTe quantum dots. Adv Mater 2006;18:1381-6.

[45] Tseng W-B, Lee C-H, Tseng W-L. Poly (diallydimethylammonium chloride)-induced dispersion and exfoliation of tungsten disulfide for the sensing of glutathione and catalytic hydrogenation of p-Nitrophenol. ACS Appl Nano Mater 2018;1:6808-17.

[46] Rodrigues GH, Miyazaki CM, Rubira RJ, Constantino CJ Ferreira M. Layer-by-layer films of graphene nanoplatelets and gold nanoparticles for methyl parathion sensing. ACS Appl Nano Mater 2019;2:1082-91.

[47] Qiao Y, Yao Z, Ge W, Zhang L, Wu H-C. Rapid and visual detection of heparin based on the disassembly of 
polyelectrolyte-induced pyrene excimers. Org Biomol Chem 2017;15:2569-74.

[48] Yang Z, Fan X, Cheng W, Ding Y, Zhang W. AIE nanoassemblies for discrimination of glycosaminoglycans and heparin quality control. Anal Chem 2019;91:10295-301.

[49] Ho HA, Boissinot M, Bergeron MG, Corbeil G, Doré K, Boudreau D, et al. Colorimetric and fluorometric detection of nucleic acids using cationic polythiophene derivatives. Angew Chem Int Ed 2002;114:1618-21.

[50] Zhan R, Fang Z, Liu B. Naked-eye detection and quantification of heparin in serum with a cationic polythiophene. Anal Chem 2010;82:1326-33.

[51] Sommers CD, Mans DJ, Mecker LC, Keire DA. Sensitive detection of oversulfated chondroitin sulfate in heparin sodium or crude heparin with a colorimetric microplate based assay. Anal Chem 2011;83:3422-30.

[52] Sommers CD, Keire DA. Detection of possible economically motivated adulterants in heparin sodium and low molecular weight heparins with a colorimetric microplate based assay. Anal Chem 2011;83:7102-8.
[53] Toby TK, Sommers CD, Keire DA. Detection of native chondroitin sulfate impurities in heparin sodium with a colorimetric micro-plate based assay. Anal Methods 2012;4: 1488-91.

[54] Wang W, Han N, Li R, Han W, Zhang X, Li F. Supercharged fluorescent protein as a versatile probe for the detection of glycosaminoglycans in vitro and in vivo. Anal Chem 2015;87: 93027.

[55] Aich U, Shriver Z, Tharakaraman K, Raman R, Sasisekharan R. Competitive inhibition of heparinase by persulfonated glycosaminoglycans: a tool to detect heparin contamination. Anal Chem 2011;83:7815-22.

[56] Kalita M, Balivada S, Swarup VP, Mencio C, Raman K, Desai UR, et al. A nanosensor for ultrasensitive detection of oversulfated chondroitin sulfate contaminant in heparin. J Am Chem Soc 2014;136:554-7.

[57] Ding Y, Zhou M, Wei H. A supercharged fluorescent protein based FRET sensing platform for detection of heparin contamination. Anal Methods 2017;9:5593-7. 